\title{
Josephson junctions in double nanowires bridged by in-situ deposited superconductors
}

\author{
Alexandros Vekris $\odot,{ }^{1,2}$ Juan Carlos Estrada Saldaña $\odot,{ }^{1}$ Thomas Kanne $\odot,{ }^{1}$ Mikelis Marnauza $\odot,{ }^{1}$ Dags Olsteins, ${ }^{1}$ \\ Furong Fan $\odot,{ }^{3}$ Xiaobo Li, ${ }^{3}$ Thor Hvid-Olsen $\odot,{ }^{1}$ Xiaohui Qiu, ${ }^{4,5}$ Hongqi Xu $\odot,{ }^{3,6}$ Jesper Nygård $\odot,{ }^{1}$ \\ and Kasper Grove-Rasmussen $\oplus^{1, *}$ \\ ${ }^{1}$ Center For Quantum Devices, Niels Bohr Institute, University of Copenhagen, 2100 Copenhagen, Denmark \\ ${ }^{2}$ Sino-Danish Center for Education and Research (SDC) SDC Building, Yanqihu Campus, University of Chinese Academy of Sciences, 380 \\ Huaibeizhuang, Huairou District, 101408 Beijing, China \\ ${ }^{3}$ Beijing Key Laboratory of Quantum Devices, Key Laboratory for the Physics and Chemistry of Nanodevices \\ and Department of Electronics, Peking University, Beijing 100871, China \\ ${ }^{4}$ CAS Key Laboratory of Standardization and Measurement for Nanotechnology, National Center for Nanoscience \\ and Technology, Beijing 100190, China \\ ${ }^{5}$ CAS Center for Excellence in Nanoscience, National Center for Nanoscience and Technology, Beijing 100190, China \\ ${ }^{6}$ Beijing Academy of Quantum Information Sciences, 100193 Beijing, China
}

(Received 5 May 2021; revised 23 July 2021; accepted 10 August 2021; published 10 September 2021)

\begin{abstract}
We characterize parallel double quantum dot Josephson junctions based on closely spaced double nanowires bridged by in-situ deposited superconductors. The parallel double dot behavior occurs despite the proximity of the two nanowires and the potential risk of nanowire clamping during growth. By tuning the charge filling and lead couplings, we map out the parallel double quantum dot Yu-Shiba-Rusinov phase diagram. Our quasiindependent two-wire hybrids show promise for the realization of exotic topological phases.
\end{abstract}

DOI: 10.1103/PhysRevResearch.3.033240

\section{INTRODUCTION}

Double Rashba-nanowires bridged by superconductors are at the center of proposals for qubits [1], coupled subgap states [2], and exotic topological superconducting phases based on Majorana zero modes (MZMs) [3-16]. Researchers have theorized on the existence of a topological Kondo phase in such wires when the bridging superconductor is in Coulomb blockade $[3,4,15,17]$ and, more recently, described a device hosting parafermions [6]. Realization of these proposals should benefit from material science developments, resulting in improved nanowire-superconductor interfaces with low quasiparticle poisoning rates [18-20].

These clean interfaces have been used in the pursuit of MZMs in single nanowires [19,21] and, more recently, for coupling single and serial quantum dots (QDs) defined on single nanowires to superconductors to realize one and two-impurity Yu-Shiba-Rusinov (YSR) models [22-26]. YSR states, belonging to the class of Andreev bound states [23-25,27-39], arise in the limit of large Coulomb charging energy, $U>\Delta$, as a result of the virtual excitation of a quasiparticle into the edge of the superconducting gap [40,41]. This quasiparticle can exchange-fluctuate with a localized spin in

\footnotetext{
*k_grove@nbi.ku.dk

Published by the American Physical Society under the terms of the Creative Commons Attribution 4.0 International license. Further distribution of this work must maintain attribution to the author(s) and the published article's title, journal citation, and DOI.
}

the QD, and if the exchange coupling is strong (i.e., when the Kondo temperature, $T_{\mathrm{K}}$, is larger than $\sim 0.3 \Delta$ ), the ground state (GS) changes from a doublet to a singlet [42]. In Josephson junctions (JJs), this induces a $\pi-O$ phase-shift change in the superconducting phase difference [22,23,43-59].

Devices which use pairs of QDs placed in a parallel configuration [60-62] and coupled to common superconducting leads have been extensively studied with the purpose of producing entangled electron states through Cooper pair splitting [63-66]. However, the behavior of the switching current, $I_{\mathrm{sw}}$, in the presence of YSR screening $[23,37,67]$ in parallel double QDs remains to be investigated.

In this paper, we characterize superconductivity in closely spaced pairs of InAs nanowires bridged by a thin epitaxial superconducting aluminum film deposited in situ [68]. To do so, we fabricate two side-by-side JJs out of one pair of nanowires and demonstrate that each nanowire hosts a single QD, through which supercurrent flows. From the charge stability diagram and magnetic field measurements, we establish that the interwire tunneling at the junction is negligible with an upper bound of $\sim 50 \mu \mathrm{eV}$. The YSR physics is analyzed through the gate dependence of the linear conductance and $I_{\text {sw }}$, where we find that the common superconducting leads screen individually each QD, hinting at individual YSR clouds instead of a single one extending over the two QDs. We furthermore show indications of supercurrent interference when the GS parities of the QDs are different, reminiscent of a superconducting quantum interference device (SQUID) at zero magnetic field.

The paper is structured in sections. In Sec. II, we introduce the YSR double QD phase diagram and measurements 
TABLE I. Parameters for shells $\mathrm{W}, \mathrm{X}, \mathrm{Y}$ of device 1 . The charging energies, $U_{\mathrm{L}, \mathrm{R}}$, are extracted from Coulomb diamond spectroscopy. The total tunneling rates of each QD, $\Gamma_{\mathrm{L}, \mathrm{R}}$, are obtained by (a) fitting the even side of Coulomb diamonds in the normal state or (b) from the full width at half maximum of the corresponding Coulomb peak. The Kondo temperature, $T_{K}$, is obtained by (c) fitting the Kondo peak (when applicable) or by (d) using the equation $T_{K}=\frac{1}{2 k_{B}} \sqrt{\Gamma U} e^{\pi \epsilon_{0}\left(\epsilon_{0}+U\right) / \Gamma U}$, with $\Gamma_{\mathrm{L}, \mathrm{R}}, U_{\mathrm{L}, \mathrm{R}}$ as known values, and $\epsilon_{0}=\epsilon_{\mathrm{L}, \mathrm{R}}$ the level position of the corresponding QD. Extraction methods are presented in detail in SM, Sec. III. From the charge stability diagram, we extract similar side-gate and back-gate capacitances for the left and right QD in the order of $C_{\mathrm{g}_{\mathrm{L}}, \mathrm{g}_{\mathrm{R}}, \mathrm{bg}} \sim 1 \mathrm{aF}$ and thus the charging energies are dominated by the source and drain capacitances.

\begin{tabular}{|c|c|c|c|c|c|c|c|c|c|c|}
\hline Shell & $U_{\mathrm{L}}(\mathrm{meV})$ & $U_{\mathrm{R}}(\mathrm{meV})$ & $\Gamma_{\mathrm{L}}(\mathrm{meV})$ & $\Gamma_{\mathrm{R}}(\mathrm{meV})$ & $\frac{\Gamma_{\mathrm{L}}}{U_{\mathrm{L}}}$ & $\frac{\Gamma_{\mathrm{R}}}{U_{\mathrm{R}}}$ & $k_{B} T_{K_{\mathrm{L}}}(\mathrm{meV})$ & $k_{B} T_{K_{\mathrm{R}}}(\mathrm{meV})$ & $\frac{k_{B} T_{K_{\mathrm{L}}}}{0.3 \Delta}$ & $\frac{k_{B} T_{K_{\mathrm{R}}}}{0.3 \Delta}$ \\
\hline W & $3.8 \pm 0.5$ & $2.3 \pm 0.3$ & $0.23 \pm 0.02^{a}$ & $0.6 \pm 0.1^{b}$ & $0.06 \pm 0.01$ & $0.26 \pm 0.05$ & $(3.1 \pm 0.3) \times 10^{-5^{d}}$ & $0.03 \pm 0.01^{d}$ & $6 \times 10^{-4}$ & 0.5 \\
\hline$X$ & $3.7 \pm 0.5$ & $1.1 \pm 0.3$ & $0.33 \pm 0.01^{a}$ & $0.55 \pm 0.05^{d}$ & $0.09 \pm 0.01$ & $0.5 \pm 0.1$ & $(8 \pm 1) \times 10^{-5^{d}}$ & $0.07-0.18^{c, \mathrm{a}}$ & 0.001 & 3.2 \\
\hline Y & $3.6 \pm 0.5$ & $1.1 \pm 0.3$ & $1.05 \pm 0.01^{a}$ & $0.55 \pm 0.05^{d}$ & $0.29 \pm 0.04$ & $0.5 \pm 0.1$ & $0.06 \pm 0.02^{d}$ & $0.07-0.18^{c}$ & 1 & 3.2 \\
\hline
\end{tabular}

a Using method (d), we extract $0.06 \mathrm{meV}$.

of two double QD shells in different coupling regimes are presented, establishing weak interdot coupling. In Sec. III, we show signatures of interference between the supercurrents flowing through each junction. In Sec. IV, we demonstrate the YSR screening evolution of $I_{\text {sw }}$. Finally, in Sec. V we present our conclusions and provide perspectives of our paper.

\section{CHARACTERIZATION OF THE PARALLEL QUANTUM-DOT JOSEPHSON JUNCTION}

In this section, we outline the device layout and demonstrate the Josephson effect and weak interdot tunneling in device 1. Data from an additional device (device 2) is shown in the Supplemental Material (SM) [69,70].

Figure 1(a) shows a falsely colored scanning electron microscopy (SEM) image of device 1. Two 80-nm InAs nanowires (in green), grown close to each other in a molecular beam epitaxy chamber and each covered on three of its facets by an in-situ deposited 17-nm-thick layer of aluminum (in blue) [68], are individually picked with a micromanipulator and deposited on a $\mathrm{Si} / \mathrm{SiOx}$ substrate with an oxide thickness of $275 \mathrm{~nm}$. A resist mask is defined by electron beam lithography to selectively etch $\mathrm{Al}$ using the commercial etchant Transene-D, creating a parallel double JJ with $\approx 100$ nm-wide bare sections of the two nanowires as weak links. $\mathrm{Ti} / \mathrm{Au} 5 \mathrm{~nm} / 250$-nm-thick contacts and individual nanowire side gates are deposited after a subsequent lithography step. Prior to the metal deposition step and without breaking vacuum, the Al native oxide is removed by argon milling to establish a good contact between $\mathrm{Ti} / \mathrm{Au}$ and $\mathrm{Al}$. The devices are measured in a dilution refrigerator at base temperature $T=30 \mathrm{mK}$.

QDs are formed when the two nanowires are brought near depletion with the use of the individual side-gate voltages, $V_{\mathrm{gL}}$ and $V_{\mathrm{gR}}$. The side gates are also used as plunger gates of the QDs. A global back gate $V_{\mathrm{bg}}$ is used to tune the coupling between the contacts and the QDs, allowing us to explore different coupling regimes. The combination of side gates and back gate also makes it easier to obtain a double QD shell structure. In Fig. 1(b), we sketch the tunneling rates of the QDs to the common superconducting leads (SC), $\Gamma_{\mathrm{L} 1}, \Gamma_{\mathrm{L} 2}$, $\Gamma_{\mathrm{R} 1}$, and $\Gamma_{\mathrm{R} 2}$, which may vary among different shells of the QDs and can be tuned by $V_{\mathrm{bg}}$. The QDs may also be coupled to each other by an interdot tunnel coupling, $t_{\mathrm{d}}$. We identify the different shells by the letters W, X, Y. QD parameters extracted for these are given in Table I. For an overview of the different shells explored, see SM, Sec. I.

The source and the drain contacts of the device each branch out into two leads as shown in Fig. 1(a), enabling us to characterize the parallel $\mathrm{JJ}_{\mathrm{S}}$ [71] in a four-terminal configuration (at the level of the leads) by applying a current, $I_{\text {bias }}$, from source to drain leads and measuring the voltage response, $V$, in a different pair of leads. In this way, we obtain $I_{\text {bias }}-V$ curves which switch from a supercurrent branch at low $I_{\text {bias }}$ to a high-slope dissipative branch at $I_{\mathrm{sw}}$. Two such curves are shown in Figs. 1(c) and 1(d) for the open and Coulomb blockaded regimes, respectively. We measure $I_{\mathrm{sw}}$ up to $35 \mathrm{nA}$ in the former regime and up to approximately $500 \mathrm{pA}$ in the latter regime. Figure $1(\mathrm{~d})$ is measured with $\mathrm{QD}_{\mathrm{L}}$ in Coulomb blockade and $\mathrm{QD}_{\mathrm{R}}$ near a Coulomb resonance. Note that the supercurrent exhibits hysteresis, as the switching is found at different currents for positive and negative applied bias. In the Coulomb blockade, the supercurrent branch shows a finite slope, $R_{\mathrm{S}}$, which increases with $\sim 1 / I_{\mathrm{sw}}$; however, this does not affect our identification of $I_{\mathrm{sw}}$ as a jump in the curve down to $5 \mathrm{pA}$ (see SM, Sec. II). In our analysis below (Sec. III), we do not claim quantitative estimates of the critical current, $I_{\mathrm{c}}$ (which may be larger), but merely address the qualitative behavior of $I_{\mathrm{sw}}$. From independent $I_{\text {bias }}-V$ measurements in the open regime, we estimate an upper bound of the contact resistance between the metal-lead and the hybrid-nanowire in the order of $20 \Omega$ (see SM for discussion).

As a guide to the different GS configurations accessed in this paper, we show in Fig. 1(e) a sketch of the phase diagram of the parallel double quantum dot (DQD) JJ versus coupling to the leads when the two QDs have independent GSs $\left(t_{\mathrm{d}}=0\right)$. The sketch corresponds to odd occupancy $(1,1)$ of the QDs and it is valid for the large level-spacing regime, $\Delta E_{i}>U_{i}$, where $i$ stands for left and right QDs. The independent-GS case is applicable to our device as most $I_{\mathrm{sw}}$ measurements are done away from the triple points of the QDs, where the effect of a finite $t_{\mathrm{d}}$ is negligible. GS changes occur when the total tunneling rates $\Gamma_{L, R}$ of each of the QDs to the common superconducting leads surpass a threshold which depends on $U_{\mathrm{L}, \mathrm{R}} / \Delta$ [31], where $\Delta$ is the superconducting gap. Above this threshold, the spin of each QD is individually screened by the superconducting 


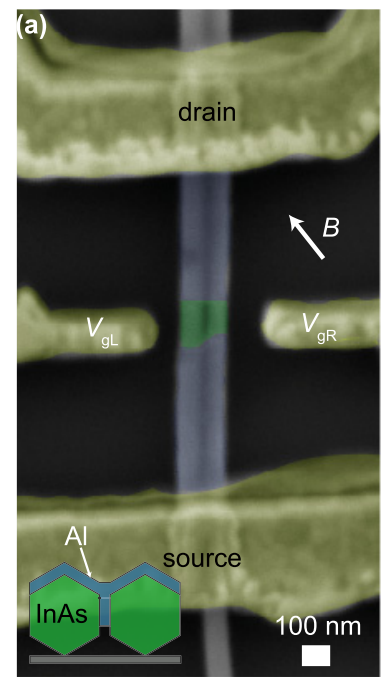

(b)
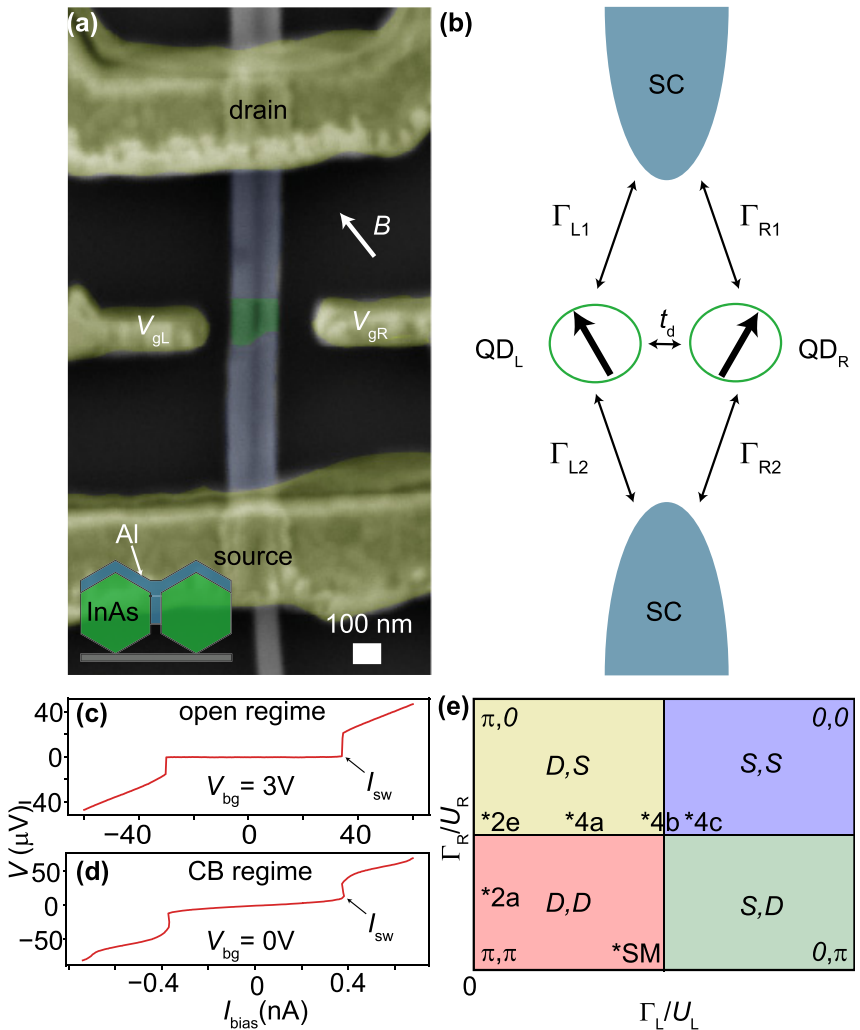

FIG. 1. (a) Scanning electron micrograph of device 1. Two nanowires with common superconducting leads form two parallel Josephson junctions. Side-by-side quantum dots serve as weak links for each JJ. The direction of an external in-plane magnetic field, $B$, when applied, is indicated by an arrow and has an angle of $45^{\circ}$ with the device. In inset, a schematic cross section of the double nanowire is shown, indicating facets of the nanowires covered by $\mathrm{Al}$ at the leads. (b) Sketch of the two side QDs coupled to two superconducting leads. Interdot tunnel coupling, $t_{\mathrm{d}}$, may be present. The GS parity of the left (L) and right (R) QDs is changed by tuning their level positions, $\epsilon_{\mathrm{L}}$ and $\epsilon_{\mathrm{R}}$, or by increasing the total tunneling rates of each QD to the leads, $\Gamma_{\mathrm{L}}=\Gamma_{\mathrm{L} 1}+\Gamma_{\mathrm{L} 2}$ and $\Gamma_{\mathrm{R}}=\Gamma_{\mathrm{R} 1}+\Gamma_{\mathrm{R} 2}$. (c,d) $I_{\text {bias }}-V$ curves measured at $V_{\mathrm{bg}}=3 \mathrm{~V}$ and $V_{\mathrm{bg}}=0 \mathrm{~V}$ showing switching current, $I_{\mathrm{sw}}$, in the open and in the Coulomb blockaded regimes, respectively. $I_{\text {bias }}$ is swept from negative to positive. (e) Sketch of the GS phase diagram depending on the tunneling rates $\Gamma_{i}$ $(i=\mathrm{L}, \mathrm{R})$ between the leads and the two QDs, when $t_{\mathrm{d}}=0$ and each QD has an unpaired electron. $D$ stands for doublet and $S$ for singlet. The expected phase shift in the Josephson current-phase relationship of each QD JJ, 0 or $\pi$, is indicated. The qualitative $\Gamma_{\mathrm{L}}, \Gamma_{\mathrm{R}}$ positions of different shells from Figs. 2, 4 and SM, Sec. VI (device 2) are indicated by asterisks.

leads via the YSR mechanism [2,72]. For a doublet GS, the current-phase relationship is $\pi$ shifted, e.g., $I=I_{\mathrm{c}} \sin (\phi+\pi)$ $[45,47,49,54]$, as indicated in Fig. 1(e). The simple analysis above is valid when a single-level Coulomb-blockaded QD acts as a weak link instead of, e.g., a quantum point contact (single barrier), where a nonsinusoidal current-phase relationship applies [73].

To estimate $t_{\mathrm{d}}$, we first investigate via two-terminal voltage-biased differential conductance $(d I / d V)$ measurements two shells corresponding to the two leftmost quadrants
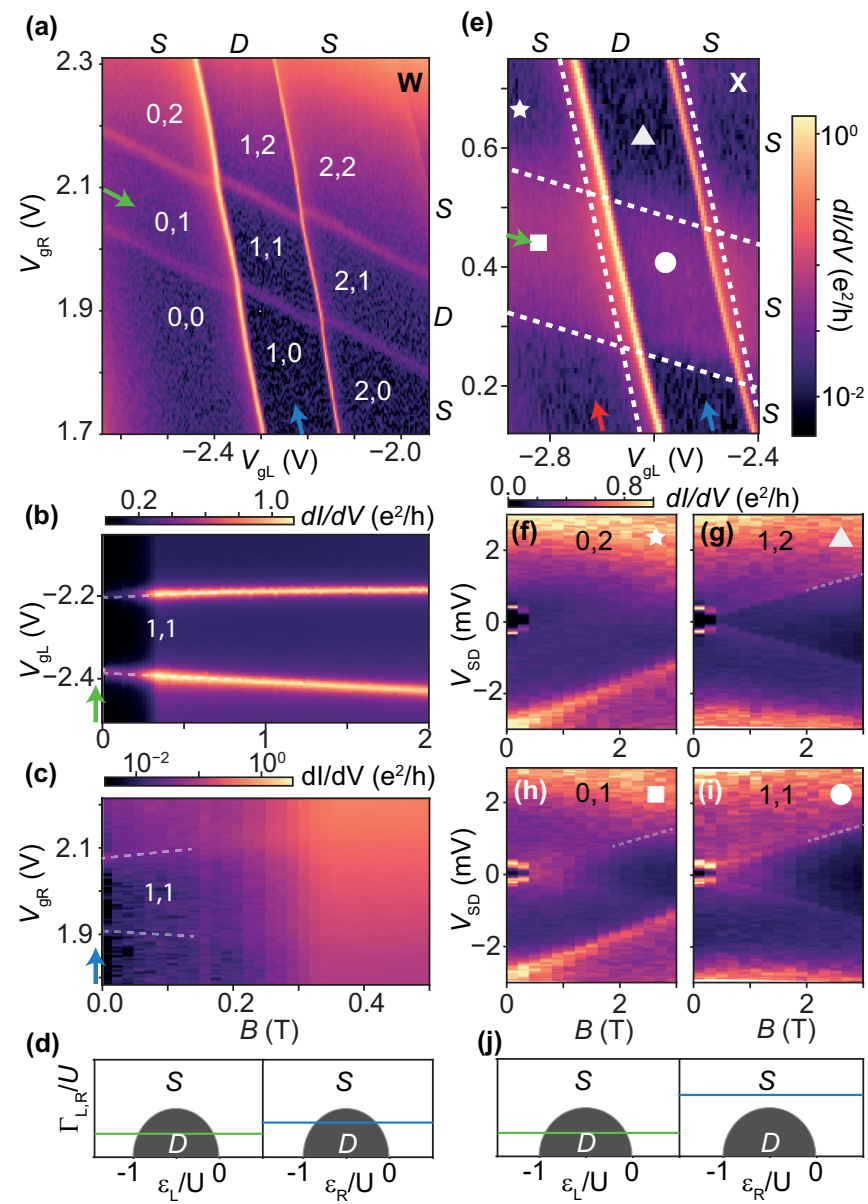

FIG. 2. (a), (e) Color maps of two-terminal, voltage-biased zerobias differential conductance, $d I / d V$, in the superconducting state for shells W (a) and X (e) versus left and right QD plunger gates. In (a), charges $N_{\mathrm{L}}, N_{\mathrm{R}}$ correspond to the charge occupation of the highest unoccupied energy level of each QD. In (e), white dashed lines represent the position of the Coulomb lines measured at $B=2 \mathrm{~T}$. (b), (c) Zero-bias $d I / d V$ color maps showing the magnetic field, $B$, dependence of parity transition lines which enclose the 1,1 charge sector in (a) versus plunger gate voltages of the (b) left and (c) right QDs, obtained by sweeping the gates along the green and blue arrows, shown in (a). For simplicity, only $V_{\mathrm{gL}}$ and $V_{\mathrm{gR}}$ are, respectively, shown. (f)-(i) Color maps of $d I / d V$ versus magnetic field, $B$, and source-drain bias voltage, $V_{\mathrm{sd}}$, taken in four different charge sectors indicated by symbols in (e). Higher $B$ field measurement of (h) can be found in SM Sec. III. Dashed lines are added as a guide to the eye. (d), (j) Pairs of phase-diagram sketches for independent left and right QDs. Horizontal color-coded lines in each pair indicate qualitatively $\Gamma_{\mathrm{L}}\left(\Gamma_{\mathrm{R}}\right)$ versus left (right) QD level position $\epsilon_{\mathrm{L}}\left(\epsilon_{\mathrm{R}}\right)$ in the stability diagrams of (a) and (e), respectively, following the arrows shown.

of the DQD phase diagram in Fig. 1(e). The two-terminal $d I / d V$ is recorded using standard lock-in amplifier techniques with an $\mathrm{AC}$ excitation of $2 \mu \mathrm{V}$. Figure 2(a) shows a color map of $d I / d V$ at source-drain bias $V_{\mathrm{SD}}=0$ of shell $\mathrm{W}$ in the superconducting state versus $V_{\mathrm{gL}}$ and $V_{\mathrm{gR}}$, which represents the stability diagram of the two QDs in the weaklycoupled regime where $\Gamma_{\mathrm{L}, \mathrm{R}} \ll U_{\mathrm{L}, \mathrm{R}}$ (see Table I for shell parameters). Since the slope of the supercurrent branch, $R_{\mathrm{S}}$, is empirically related in our device to $1 / I_{\mathrm{sw}}$, we can use 
$R_{\mathrm{S}}=1 /\left(d I / d V\left(V_{\mathrm{SD}}=0\right)\right)$ as an indicator of the magnitude of $I_{\mathrm{sw}}$. This is particularly relevant in the Coulomb-blockade regime, when $I_{\mathrm{sw}}$ is small and $R_{\mathrm{S}}$ is significant (see $\mathrm{SM}$, Sec. II). We only use this empirical relation to comment on the voltage-biased measurements in Fig. 2. We observe approximately vertical and horizontal conductance lines which overlap and displace each other at their crossings, without exhibiting any significant bending. The displacement is a signature of a finite interdot charging energy, while the lack of bending indicates that $t_{\mathrm{d}} \approx 0$ (with an upper limit of $50 \mu \mathrm{V}$ based on the width of the sharpest conductance lines). No signatures of crossed-Andreev reflection (CAR) or of elastic cotunneling [74] are observed in this measurement. We interpret these lines as GS parity transition lines, which indicate changes of parity in the left and right QDs, respectively. The lines separate nine different and well-defined parity sectors. We assign corresponding effective left and right QD charges, $N_{\mathrm{L}}, N_{\mathrm{R}}$, to each of these sectors based on the shell-filling pattern of the stability diagram in larger plunger-gate ranges (see $\mathrm{SM}$, Sec. I). The charges obtained in this way are indicated in Fig. 2(a). These charges correspond to the charge occupation of the highest unoccupied energy level of each QD.

To assign GS parities to these nine sectors, and to determine independently if, in addition to interdot charging energy, there is a significant $t_{\mathrm{d}}$, we trace the evolution of the parity transition lines of the 1,1 charge sector against $B$. In the case of the singlet GS, i.e., when the spins of the two QDs are exchange-coupled (finite $t_{\mathrm{d}}$ ), these lines are expected to come together with $B$ [75]. Instead, as shown in the zero-bias $d I / d V$ color maps in Figs. 2(b) and 2(c), the parity transition lines enclosing the 1,1 charge sector split apart with $B$, i.e., the two QDs are independent doublets, despite the relative proximity of the two nanowires. The splitting of the parity lines occurs both in the case when the parity of the left (right) QD is varied and the right (left) QD is kept in the doublet GS [see green and blue arrow, respectively, in Fig. 2(a)]. The GS (singlet $S$ or doublet $D$ ) of the other eight charge sectors are indicated on the top and right exterior parts of the stability-diagram color map in Fig. 2(a).

Given the decoupling between the two QDs, we can approximate their phase diagrams by those of two independent single QDs. Neglecting the interdot charging energy, we sketch in Fig. 2(d) the well-known single-QD phase diagrams for the GS of the left and right QDs versus QD level position, $\epsilon_{\mathrm{L}, \mathrm{R}}$, and versus the total tunneling rate of each QD to the leads, $\Gamma_{\mathrm{L}, \mathrm{R}}$, over their charging energy, $U_{\mathrm{L}, \mathrm{R}}$. The doublet dome has an upper height limit of $\Gamma_{\mathrm{L}, \mathrm{R}} / U_{\mathrm{L}, \mathrm{R}}=1 / 2$ in the infinite $\Delta$ limit, and its height decreases in the $U \gg \Delta$ limit (i.e., the YSR regime) to which our QDs belong $[34,76]$. In the left phase diagram, the horizontal green line which crosses the doublet dome indicates a cut where $\epsilon_{\mathrm{L}}$ is varied and $\epsilon_{\mathrm{R}}$ is kept fixed such that the GS parity of the right QD is a doublet, and the GS parity of the left QD is variable. This line represents schematically the gate trajectory in Fig. 2(b), as indicated with the green arrow, which is collinear to the green arrow in Fig. 2(a), and which varies the parity of the left QD as $S-D-S$ while keeping the parity of the right QD as $D$. A similar relation exists between the horizontal blue line in the right phase diagram and the gate trajectory (blue arrow) in Fig. 2(c), also collinear to the corresponding arrow in Fig. 2(a). From these phase diagrams, we note that parity transitions are strictly equal to Coulomb degeneracies only at zero $\Gamma_{\mathrm{L}, \mathrm{R}}$. The measurements above confirm the expected DQD behavior for low lead couplings, which shows a $D, D$ ground for charge state 1,1 corresponding to the lower left quadrant of the phase diagram in Fig. 1(e).

Next, we investigate a shell with different couplings to the leads (shell $\mathrm{X}$ ) which belongs to the upper left quadrant of phase diagram in Fig. 1(e). Figure 2(e) shows the zero-bias $d I / d V$ color map in the superconducting state versus the plunger gates of the two QDs of shell X. The two horizontal GS-parity transition lines, which bounded the green trajectory in the case of shell $\mathrm{W}$, are absent in the case of shell X, and are instead replaced by a band of enhanced conductance. The conductance band is cut two times by approximately vertical conductance lines, which correspond to GS-parity transition lines of the left QD.

The parity of the band of enhanced conductance in the stability diagram is determined from the $B$ evolution of the differential conductance in the normal state versus $\mathrm{V}_{\text {sd }}$ at two fixed gate voltages. These two gate voltages are indicated by a square (charge states 0,1$)$ and a circle $(1,1)$ in the stability diagram, and their $B$ dependence is, respectively, shown in Figs. 2(h) and 2(i). As a control experiment, the $B$ dependence for two fixed gate voltages above the conductance band indicated by a star $(0,2)$ and a triangle $(1,2)$ in the stability diagram, is shown in Figs. 2(f) and 2(g). The four measurements show closing of the superconducting gap at $B=0.4 \mathrm{~T}$, which is consistent with the jump in the zero-bias $d I / d V$ signal in Figs. 2(b) and 2(c) at $B \approx 0.4 \mathrm{~T}$. However, whereas Figs. 2(g)-2(i) $(1,20,11,1)$ display conductance steps near zero bias which split with $B$ field in the normal state, there is no such splitting in Fig. 2(f), consistent with even filling of both dots. We assign effective QD charge numbers to the charge stability diagram from a $B=2 \mathrm{~T}$ measurement (see SM, Sec. III) and overlay the Coulomb lines obtained, which delimit the nine charge sectors [white dashed lines in Fig. 2(e)].

We note an additional important difference in the data of the low-bias splitting states. In Fig. 2(g) $(1,2)$, the splitting can be traced back to zero bias at $B=0$, while in Fig. 2(h) $(0,1)$ the splitting is traced to zero bias only at a finite field of $\approx 1 \mathrm{~T}$. The pair of features whose splitting can be traced to a $B=0$ onset in Fig. 2(g) $(1,2)$ correspond to cotunneling steps of the odd-occupied left QD experiencing Zeeman splitting. In turn, the pair of features which starts to split at $1 \mathrm{~T}$ in Fig. 2(h) corresponds to the Zeeman splitting of a Kondo resonance in the right QD. The splitting ensues when $E_{\mathrm{Z}} \sim k_{B} T_{K_{\mathrm{R}}}$ [77]. Notice that the Kondo resonance is also visible in the data after the gap closure at $B=0.4 \mathrm{~T}$. From the splitting, we find a $g$-factor $g \sim 8.5 \pm 0.1$. Table I shows that $k_{B} T_{K_{\mathrm{R}}}>0.3 \Delta$ for shell $\mathrm{X}$, which is consistent with a YSR singlet state in the right $\mathrm{QD}$ in the superconducting state.

The $B$-dependence data in Figs. 2(f)-2(i) therefore allows us to assign the GS to the QDs, $D$ or $S$, in each of the nine sectors in Fig. 2(e). We indicate schematically by a green and blue horizontal line in the two individual-QD phase diagrams in Fig. 2(j) the GS along the gate trajectories collinear to the same-colored arrows in the color map of Fig. 2(e). The green (blue) gate trajectory, which goes along (perpendicular to) the 
band of enhanced conductance intersects twice (goes above) the doublet dome, leading to two (zero) parity transitions.

\section{SUPERCURRENT INTERFERENCE FOR DIFFERENT QUANTUM-DOT PARITIES}

We switch back to the four-terminal measurement configuration to correlate the intrinsic phase of each $\mathrm{JJ}$ with the magnitude of $I_{\mathrm{sw}}$. In Fig. 3, we show $I_{\mathrm{sw}}$ versus plunger gate voltages, where $I_{\mathrm{sw}}$ is extracted in a similar fashion as in Fig. 1(d). In Figs. 3(a) and 3(c) [Figs. 3(b) and 3(d)], the plunger gate voltages are swept along trajectories which vary the occupation in the left (right) QD while keeping the occupation of the right (left) QD fixed, following the green (red, blue) arrows in Figs. 2(a) and 2(e), i.e., for shells W and $\mathrm{X}$, respectively. For reference, we assign the expected phase shift in the current-phase relationship, $\pi$ or 0 , based on the measured GS parities of the two QDs. This phase shift is accurate when at least one QD is in Coulomb blockade. The value of $I_{\mathrm{sw}}$ at the parity transitions may include a contribution due to the presence of bound states crossing zero energy. Hence, the magnitude of $I_{\mathrm{sw}}$ on transitions should not be taken into account.

The common phenomenology in the data is as follows. After a smooth buildup of $I_{\mathrm{sw}}$ toward a $O \rightarrow \pi$ transition, the current abruptly drops at the edge of the $\pi$ domain, resulting in an asymmetric $I_{\text {sw }}$ peak [49]. A pair of asymmetric peaks is seen in the data in Figs. 3(a)-3(c), as one of the QDs experiences parity transitions and therefore a sequence of $0-\pi-0$ phase-shift changes. If the parity stays unchanged, such peaks are absent, as in Fig. 3(d). Instead, $I_{\text {sw }}$ is smoothly
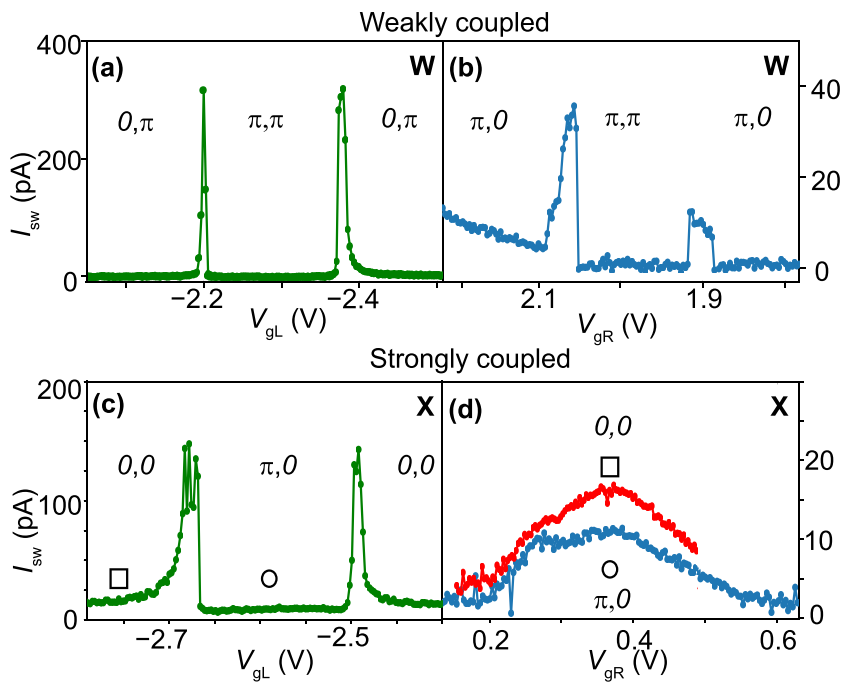

FIG. 3. (a)-(d) Extracted $I_{\mathrm{sw}}$ versus plunger-gate voltage trajectories collinear to same-colored arrows in [(a), (b)] Fig. 2(a), shell W, and (c), (d) Fig. 2(e), shell X. In (d), two traces are shown to illustrate the decrease in $I_{\mathrm{sw}}$ as a consequence of the subtracting effect of a $\pi$ phase shift in one of the QD Josephson junctions. The red curve is offset on the gate axis to correct for the cross talk between the gates and the QDs. The $I_{\mathrm{sw}}$ is extracted by measuring the $I_{\text {bias }}-V$ curve from negative to positive current for each gate value, and is identified as the switching on the positive current branch. enhanced toward odd occupation of the right $\mathrm{QD}$, which is YSR screened (i.e., $k_{B} T_{K}>0.3 \Delta$ ) [54]. Interestingly, when comparing the red and blue traces in Fig. 3(d), which correspond to different phase shifts ( $\pi$ and 0 , respectively) in the $\mathrm{JJ}$ formed by the left QD, we observe that $I_{\mathrm{sw}}$ is stronger near $V_{\mathrm{gR}}=0.4 \mathrm{~V}$. Note that $V_{\mathrm{gR}}=0.4 \mathrm{~V}$ corresponds to the 1,1 charge state for the blue trace, and to the 0,1 charge state for the red trace. The exact magnitude of $I_{\mathrm{sw}}$ in that gate value for the red and blue curves is consistent with what is found in Fig. 3(c) in the ( $\square$ ) and ( $\bigcirc$ ), respectively. We can interpret the reduction in $I_{\mathrm{sw}}$ at $V_{\mathrm{gR}}=0.4 \mathrm{~V}$ in the blue trace with respect to the red trace by considering the double nanowire device as a SQUID at zero threaded magnetic flux $[45,47,54]$. The $I_{c}$ of a SQUID with a sinusoidal current-phase relation at zero flux can be written as [54]

$$
I_{\mathrm{c}}=\sqrt{\left(I_{\mathrm{c} 1}-I_{\mathrm{c} 2}\right)^{2}+4 I_{\mathrm{c} 1} I_{\mathrm{c} 2}\left|\cos \left(\frac{\delta_{1}+\delta_{2}}{2}\right)\right|^{2}},
$$

where $I_{\mathrm{c} 1, \mathrm{c} 2}$ are the critical currents of the two JJs and $\delta_{1,2}$ are the intrinsic phase shifts $(0$ or $\pi)$ of the junctions. As a result, the total $I_{\mathrm{c}}$ is given by $I_{\mathrm{c}} \square=I_{\mathrm{c} 1}+I_{\mathrm{c} 2}$ when the DQD is in the 0,0 phase and $I_{\mathrm{c}} \bigcirc=I_{\mathrm{c} 1}-I_{\mathrm{c} 2}$ in the $\pi, 0$ phase. These equations can explain the findings in Figs. 3(c) and 3(d), as $I_{\mathrm{SW}}$ is enhanced when both JJs have the same intrinsic phase, and it is weaker when the two JJs have different phases.

\section{SCREENING EVOLUTION OF SWITCHING CURRENT}

Finally, we demonstrate individual control of the couplings between the SC leads and the QDs, realizing the transition from the upper left (one screened spin in 1,1) to upper right quadrant (both spins screened) in the YSR phase diagram depicted in Fig. 1(e). Whereas the changes in GS parity in Fig. 2 occurred primarily by changing the side-gate voltages to go from shell $\mathrm{W}$ to shell $\mathrm{X}$, here the changes occur within a unique shell. This is done in a shell identified as $\mathrm{Y}$, using $V_{\mathrm{bg}}$ as a tuning knob of $\Gamma_{\mathrm{L}, \mathrm{R}}$. In Figs. 4(a)-4(c), we show color maps representing parity stability diagrams at different $V_{\mathrm{bg}}$ analogous to those in Figs. 2(a) and 2(e); however, instead of plotting a measurement of voltage-biased $d I / d V$, we directly plot a four-terminal measurement of $I_{\mathrm{sw}}$ versus plunger-gate voltages. To obtain each color map, we measure the $I_{\text {bias }}-V$ characteristic at each plunger gate voltage coordinate (i.e., at each pixel in the color map) and extract $I_{\text {sw }}$ as in the example in Fig. 1(d).

In Fig. 4(a), the $I_{\text {sw }}$ parity stability diagram shows two $I_{\text {sw }}$ peaks which correspond to two parity transitions of the left QD. The lack of right-QD parity transition lines indicates that the right QD is YSR screened. We corroborate that this is indeed the case from a measurement of $T_{K_{\mathrm{R}}}$ at $B=0.4 \mathrm{~T}$ in the normal state, and we find $k_{B} T_{K_{\mathrm{R}}}>0.3 \Delta$ (see Table I). We also note that, although faintly visible here, a two-terminal $d I / d V$ measurement of the stability diagram in otherwise the same conditions as here displays an horizontal band of (weakly) enhanced conductance, which is the same phenomenology identified in Fig. 2(d) with YSR spin screening. However, the enhancement is weak enough to preclude resolution of $I_{\mathrm{sw}}$, and therefore a similar band of $I_{\mathrm{sw}}$ only shows at the right part of Fig. 4(a) $\left(\mathrm{V}_{\mathrm{gL}} \approx-2.95 \mathrm{~V}, \mathrm{~V}_{\mathrm{gR}} \approx 0.45 \mathrm{~V}\right)$. 

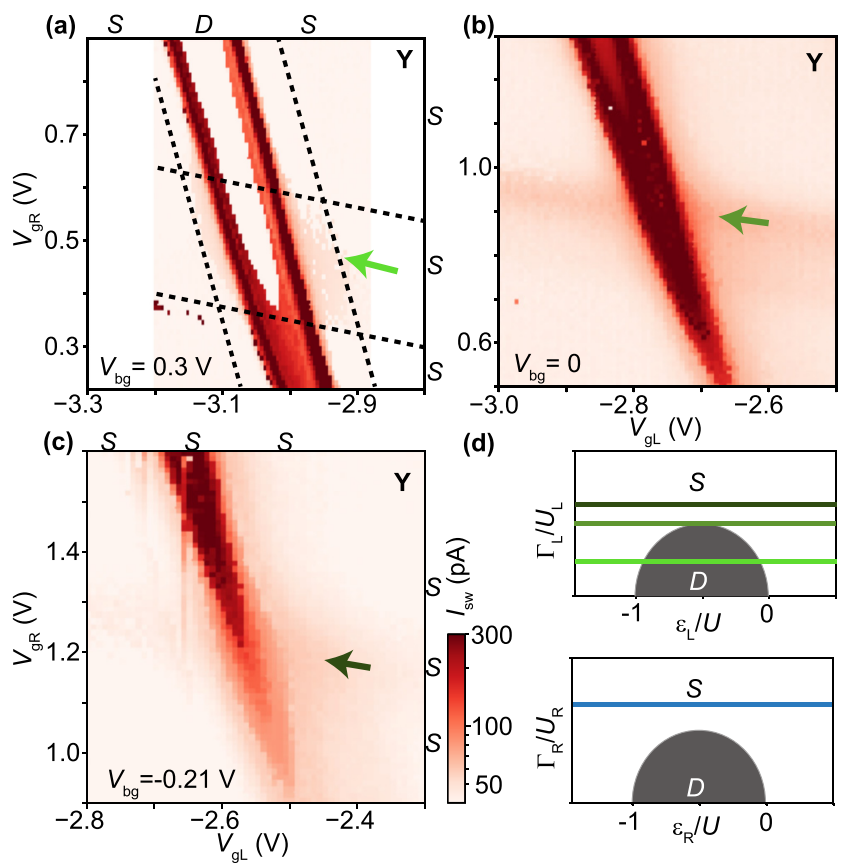

FIG. 4. (a)-(c) Color maps of $I_{\mathrm{sw}}$ as a function of the plunger gates of the two QDs, taken at three different $V_{\mathrm{bg}}$ values in shell Y. In (a), Coulomb lines positions (black dashed lines) are obtained from a normal-state two-terminal differential conductance measurement at $B=2 \mathrm{~T}$. To keep shell $\mathrm{Y}$ in frame, the effect of $V_{\mathrm{bg}}$ has been compensated by changing $V_{\mathrm{gL}}$ and $V_{\mathrm{gR}}$. In (a) and (c), the GS of the two independent QDs is indicated on the exterior side of the color maps. (d) Independent-QD phase-diagram sketches as function tunneling rate $\Gamma_{\mathrm{L}, \mathrm{R}}$ and QD level position, $\epsilon_{\mathrm{L}, \mathrm{R}}$, for the left QD (top panel) and right QD (lower panel). In the top panel, green-shaded horizontal lines indicate qualitatively $\Gamma_{\mathrm{L}}$ in directions collinear to the arrows of the same color in (a)-(c). The blue line indicates qualitatively $\Gamma_{\mathrm{R}}$ in (a)-(c). Note that decreasing the back-gate voltage results in stronger coupling to the left superconducting lead. The $I_{\mathrm{sw}}$ is extracted by measuring the $I_{\text {bias }}-V$ curve from zero to positive current for each gate point.

Reducing $\mathrm{V}_{\mathrm{bg}}$ alters the $I_{\mathrm{sw}}$ parity stability diagram by bringing the two $I_{\mathrm{sw}}$ peaks (parity lines) of the left QD closer together, as shown in Fig. 4(b). Note that a faint, approximately horizontal band of $I_{\mathrm{sw}}$ is observed along the direction pointed by the dark-green arrow, which comes as a result of enhancement of $I_{\mathrm{sw}}$ due to YSR spin-screening of the right QD. In Fig. 4(c), further reduction of $\mathrm{V}_{\mathrm{bg}}$ leads to merging of the parity lines into a vertical band of $I_{\text {sw }}$ across the whole plot. At this point, the spins of both QDs are YSR screened into singlets. We have therefore traced the phase diagram shown in Fig. 1(e), where either one spin of a QD or both are screened by the YSR mechanism, triggering a phase change in the current-phase relation of the JJs. Additional data on the magnetic field dependence of this shell can be found in the SM, Sec. IV.

\section{CONCLUSIONS AND OUTLOOK}

In conclusion, we have demonstrated parallel QD JJs fabricated out of a double-nanowire platform in which the nanowires are bridged by an in-situ deposited superconductor.
We mapped out the parallel QD YSR phase diagram via conductance and switching current measurements showing the tunability of the GS of each JJ from doublet to singlet. The analysis also revealed that the nanowires are predominantly decoupled with an upper bound on the dot tunnel coupling in the order of $t_{\mathrm{d}} \leqslant 50 \mu \mathrm{eV}$ for the specific charge states studied in two devices (see SM, Sec. VI). A lower bound is hard to identify due to the lack of evident anticross in device 1 , but in device 2 the lower bound of $t_{\mathrm{d}}$ is estimated to be in the same order of magnitude as the upper bound. In general, other shells may be stronger coupled at higher gate voltages and the interdot tunnel coupling may be increased by adjusting nanowire growth parameters [68]. Finally, we showed indications of switching current addition and subtraction via appropriate choice of GSs of the two dots involving the YSR singlet state, i.e., 0,0 and $\pi, 0$ (phase difference) regimes, respectively.

The above observations of basic superconducting properties in in-situ made hybrid double nanowire material open up for more advanced experiments addressing a number of recent theoretical proposals. In parallel double-QD Cooperpair splitters $[63,78]$, the CAR mechanism responsible for the splitting is weakened by an increase in the distance between the tunneling points from the superconductor into the two QDs [79]. The proximity of the nanowires set by growth [68] and the cleanness of the Al-InAs interface may turn out to be beneficial for CAR, which is also the basis for creating coupled YSR states in these systems [2,80]. The latter is investigated in a parallel work on the same hybrid double nanowire material [68] as used in this paper [67]. The hybrid double nanowires are furthermore prime candidates for realizing several species of topological subgap states [5,6]. For finite CAR, the requirements for entering the topological regime hosting Majorana bound states have been shown to be lowered [8,12], and parafermions may be achieved in a regime where CAR dominates over local Andreev processes [6]. In superconducting islands fabricated in our hybrid double nanowires, the topological Kondo effect can be pursued [3,4,15], and in JJs as here demonstrated, nonstandard types of Andreev bound states have been predicted [14] in the topological regime. Furthermore, a $\varphi_{0}$ junction geometry can be investigated in the double nanowire platform by implementing the double nanowires in a SQUID. As an ending remark, we note that double nanowires can also be made with a full superconducting shell $[68,81]$, relevant for investigating flux-induced subgap states $[24,82,83]$.

\section{ACKNOWLEDGMENTS}

We thank Gorm Steffensen, Jens Paaske, Michele Burrello, and Constantin Schrade for useful discussions and Xinyan Wang for experimental contribution. We acknowledge the support of the Sino-Danish Center, the European Union's Horizon 2020 research and innovation program under the Marie Sklodowska-Curie Grant Agreement No. 832645, QuantERA SuperTop (NN 127900), the European Union's Horizon 2020 research and innovation programme FETOpen Grant No. 828948 (AndQC), the Danish National Research Foundation, Villum Foundation (Research Grant No. 25310), the Ministry of Science and Technology of China through the 
National Key Research and Development Program of China (Grants No. 2017YFA0303304 and No. 2016YFA0300601), the National Natural Science Foundation of China (Grant No.
11874071), the Beijing Academy of Quantum Information Sciences (Grant No. Y18G22), Carlsberg Foundation and the Independent Research Fund Denmark.
[1] D. Aasen, M. Hell, R. V. Mishmash, A. Higginbotham, J. Danon, M. Leijnse, T. S. Jespersen, J. A. Folk, C. M. Marcus, K. Flensberg, and J. Alicea, Milestones toward Majorana-Based Quantum Computing, Phys. Rev. X 6, 031016 (2016).

[2] N. Y. Yao, C. P. Moca, I. Weymann, J. D. Sau, M. D. Lukin, E. A. Demler, and G. Zaránd, Phase diagram and excitations of a Shiba molecule, Phys. Rev. B 90, 241108(R) (2014).

[3] B. Béri and N. R. Cooper, Topological Kondo Effect with Majorana Fermions, Phys. Rev. Lett. 109, 156803 (2012).

[4] A. Altland and R. Egger, Multiterminal Coulomb-Majorana Junction, Phys. Rev. Lett. 110, 196401 (2013).

[5] E. Gaidamauskas, J. Paaske, and K. Flensberg, Majorana Bound States in Two-Channel Time-Reversal-Symmetric Nanowire Systems, Phys. Rev. Lett. 112, 126402 (2014).

[6] J. Klinovaja and D. Loss, Time-reversal invariant parafermions in interacting Rashba nanowires, Phys. Rev. B 90, 045118 (2014).

[7] H. Ebisu, B. Lu, J. Klinovaja, and Y. Tanaka, Theory of timereversal topological superconductivity in double Rashba wires: Symmetries of Cooper pairs and Andreev bound states, Prog. Theor. Exp. Phys. 2016, 083 I01 (2016).

[8] C. Schrade, M. Thakurathi, C. Reeg, S. Hoffman, J. Klinovaja, and D. Loss, Low-field topological threshold in Majorana double nanowires, Phys. Rev. B 96, 035306 (2017).

[9] C. Reeg, J. Klinovaja, and D. Loss, Destructive interference of direct and crossed Andreev pairing in a system of two nanowires coupled via an $s$-wave superconductor, Phys. Rev. B 96, 081301(R) (2017).

[10] C. Schrade and L. Fu, Parity-controlled $2 \pi$ Josephson Effect Mediated by Majorana Kramers Pairs, Phys. Rev. Lett. 120, 267002 (2018).

[11] M. Thakurathi, P. Simon, I. Mandal, J. Klinovaja, and D. Loss, Majorana Kramers pairs in Rashba double nanowires with interactions and disorder, Phys. Rev. B 97, 045415 (2018).

[12] O. Dmytruk, M. Thakurathi, D. Loss, and J. Klinovaja, Majorana bound states in double nanowires with reduced Zeeman thresholds due to supercurrents, Phys. Rev. B 99, 245416 (2019).

[13] M. Thakurathi, D. Chevallier, D. Loss, and J. Klinovaja, Transport signatures of bulk topological phases in double Rashba nanowires probed by spin-polarized STM, Phys. Rev. Research 2, 023197 (2020).

[14] P. Kotetes, M. T. Mercaldo, and M. Cuoco, Synthetic Weyl Points and Chiral Anomaly in Majorana Devices With Nonstandard Andreev-Bound-State Spectra, Phys. Rev. Lett. 123, 126802 (2019).

[15] M. Papaj, Z. Zhu, and L. Fu, Multichannel charge Kondo effect and non-Fermi-liquid fixed points in conventional and topological superconductor islands, Phys. Rev. B 99, 014512 (2019).

[16] A. Haim and Y. Oreg, Time-reversal-invariant topological superconductivity in one and two dimensions, Phys. Rep. 825, 1 (2019).

[17] M. R. Galpin, A. K. Mitchell, J. Temaismithi, D. E. Logan, B. Béri, and N. R. Cooper, Conductance fingerprint of Majorana fermions in the topological Kondo effect, Phys. Rev. B 89 , 045143 (2014).

[18] P. Krogstrup, N. L. B. Ziino, W. Chang, S. M. Albrecht, M. H. Madsen, E. Johnson, J. Nygård, C. M. Marcus, and T. S. Jespersen, Epitaxy of semiconductor-superconductor nanowires, Nat. Mater. 14, 400 (2015).

[19] S. M. Albrecht, A. P. Higginbotham, M. Madsen, F. Kuemmeth, T. S. Jespersen, J. Nygård, P. Krogstrup, and C. M. Marcus, Exponential protection of zero modes in Majorana islands, Nature (London) 531, 206 (2016).

[20] A. P. Higginbotham, S. M. Albrecht, G. Kiršanskas, W. Chang, F. Kuemmeth, P. Krogstrup, T. S. Jespersen, J. Nygård, K. Flensberg, and C. M. Marcus, Parity lifetime of bound states in a proximitized semiconductor nanowire, Nat. Phys. 11, 1017 (2015).

[21] M. T. Deng, S. Vaitiekenas, E. B. Hansen, J. Danon, M. Leijnse, K. Flensberg, J. Nygård, P. Krogstrup, and C. M. Marcus, Majorana bound state in a coupled quantum-dot hybrid-nanowire system, Science 354, 1557 (2016).

[22] J. C. Estrada Saldaña, A. Vekris, G. Steffensen, R. Žitko, P. Krogstrup, J. Paaske, K. Grove-Rasmussen, and J. Nygård, Supercurrent in a Double Quantum Dot, Phys. Rev. Lett. 121, 257701 (2018).

[23] J. C. Estrada Saldaña, A. Vekris, R. Žitko, G. Steffensen, P. Krogstrup, J. Paaske, K. Grove-Rasmussen, and J. Nygård, Two-impurity Yu-Shiba-Rusinov states in coupled quantum dots, Phys. Rev. B 102, 195143 (2020).

[24] M. Valentini, F. Peñaranda, A. Hofmann, M. Brauns, R. Hauschild, P. Krogstrup, P. San-Jose, E. Prada, R. Aguado, and G. Katsaros, Non-topological zero bias peaks in full-shell nanowires induced by flux tunable Andreev states, Science 373 $82(2021)$.

[25] J. C. Estrada Saldaña, A. Vekris, V. Sosnovtseva, T. Kanne, P. Krogstrup, K. Grove-Rasmussen, and J. Nygård, Temperature induced shifts of $\mathrm{Yu}-\mathrm{Shib}-\mathrm{Rusinov}$ resonances in nanowirebased hybrid quantum dots, Commun. Phys. 3, 125 (2020).

[26] D. Razmadze, E. C. T. O'Farrell, P. Krogstrup, and C. M Marcus, Quantum Dot Parity Effects in Trivial and Topological Josephson Junctions, Phys. Rev. Lett. 125, 116803 (2020).

[27] M. R. Buitelaar, T. Nussbaumer, and C. Schönenberger, Quantum Dot in the Kondo Regime Coupled to Superconductors, Phys. Rev. Lett. 89, 256801 (2002).

[28] R. S. Deacon, Y. Tanaka, A. Oiwa, R. Sakano, K. Yoshida, K. Shibata, K. Hirakawa, and S. Tarucha, Tunneling Spectroscopy of Andreev Energy Levels in a Quantum Dot Coupled to a Superconductor, Phys. Rev. Lett. 104, 076805 (2010).

[29] R. S. Deacon, Y. Tanaka, A. Oiwa, R. Sakano, K. Yoshida, K. Shibata, K. Hirakawa, and S. Tarucha, Kondo-enhanced Andreev transport in single self-assembled InAs quantum dots contacted with normal and superconducting leads, Phys. Rev. B 81, 121308(R) (2010).

[30] J.-D. Pillet, C. H. L. Quay, P. Morfin, C. Bena, A. L. Yeyati, and P. Joyez, Andreev bound states in supercurrent-carrying carbon nanotubes revealed, Nat. Phys. 6, 965 (2010). 
[31] E. J. H. Lee, X. Jiang, M. Houzet, R. Aguado, C. M. Lieber, and S. De Franceschi, Spin-resolved Andreev levels and parity crossings in hybrid superconductor-semiconductor nanostructures, Nat. Nanotechnol. 9, 79 (2014).

[32] A. Jellinggaard, K. Grove-Rasmussen, M. H. Madsen, and J. Nygård, Tuning Yu-Shiba-Rusinov states in a quantum dot, Phys. Rev. B 94, 064520 (2016).

[33] S. Li, N. Kang, P. Caroff, and H. Q. Xu, $0-\pi$ phase transition in hybrid superconductor-InSb nanowire quantum dot devices, Phys. Rev. B 95, 014515 (2017).

[34] E. J. H. Lee, X. Jiang, R. Žitko, R. Aguado, C. M. Lieber, and S. De Franceschi, Scaling of subgap excitations in a superconductor-semiconductor nanowire quantum dot, Phys. Rev. B 95, 180502(R) (2017).

[35] Z. Su, A. B. Tacla, M. Hocevar, D. Car, S. R. Plissard, E. P. A. M. Bakkers, A. J. Daley, D. Pekker, and S. M. Frolov, Andreev molecules in semiconductor nanowire double quantum dots, Nat. Commun. 8, 585 (2017).

[36] J. Gramich, A. Baumgartner, and C. Schönenberger, Andreev bound states probed in three-terminal quantum dots, Phys. Rev. B 96, 195418 (2017).

[37] K. Grove-Rasmussen, G. Steffensen, A. Jellinggaard, M. H. Madsen, R. Žitko, J. Paaske, and J. Nygård, Yu-Shiba-Rusinov screening of spins in double quantum dots, Nat. Commun. 9, 2376 (2018).

[38] Z. Su, R. Žitko, P. Zhang, H. Wu, D. Car, S. R. Plissard, S. Gazibegovic, G. Badawy, M. Hocevar, J. Chen, E. P. A. M. Bakkers, and S. M. Frolov, Erasing odd-parity states in semiconductor quantum dots coupled to superconductors, Phys. Rev. B 101, 235315 (2020).

[39] E. Prada, P. San-Jose, M. W. A. de Moor, A. Geresdi, E. J. H. Lee, J. Klinovaja, D. Loss, J. Nygård, R. Aguado, and L. P. Kouwenhoven, From Andreev to Majorana bound states in hybrid superconductor-semiconductor nanowires, Nat. Rev. Phys. 2, 575 (2020).

[40] R. Žitko, J. S. Lim, R. López, and R. Aguado, Shiba states and zero-bias anomalies in the hybrid normal-superconductor Anderson model, Phys. Rev. B 91, 045441 (2015).

[41] G. Kiršanskas, M. Goldstein, K. Flensberg, L. I. Glazman, and J. Paaske, Yu-Shiba-Rusinov states in phase-biased superconductor-quantum dot-superconductor junctions, Phys. Rev. B 92, 235422 (2015).

[42] K. Satori, H. Shiba, O. Sakai, and Y. Shimizu, Numerical renormalization group study of magnetic impurities in superconductors, J. Phys. Soc. Jpn. 61, 3239 (1992).

[43] J. Bauer, A. Oguri, and A. C. Hewson, Spectral properties of locally correlated electrons in a Bardeen-Cooper-Schrieffer, J. Phys.: Condens. Matter 19, 486211 (2007).

[44] A. V. Rozhkov, D. P. Arovas, and F. Guinea, Josephson coupling through a quantum dot, Phys. Rev. B 64, 233301 (2001).

[45] J. A. Van Dam, Y. V. Nazarov, E. P. A. M. Bakkers, S. De Franceschi, and L. P. Kouwenhoven, Supercurrent reversal in quantum dots, Nature (London) 442, 667 (2006).

[46] F. S. Bergeret, A. L. Yeyati, and A. Martín-Rodero, Interplay between Josephson effect and magnetic interactions in double quantum dots, Phys. Rev. B 74, 132505 (2006).

[47] J.-P. Cleuziou, W. Wernsdorfer, V. Bouchiat, T. Ondarçuhu, and M. Monthioux, Carbon nanotube superconducting quantum interference device, Nat. Nanotechnol. 1, 53 (2006).
[48] K. Grove-Rasmussen, H. I. Jørgensen, and P. E. Lindelof, Kondo resonance enhanced supercurrent in single wall carbon nanotube Josephson junctions, New J. Phys. 9, 124 (2007).

[49] H. I. Jørgensen, T. Novotný, K. Grove-Rasmussen, K. Flensberg, and P. E. Lindelof, Critical current $0-\pi$ transition in designed Josephson quantum dot junctions, Nano Lett. 7, 2441 (2007).

[50] A. Eichler, R. Deblock, M. Weiss, C. Karrasch, V. Meden, C. Schönenberger, and H. Bouchiat, Tuning the Josephson current in carbon nanotubes with the Kondo effect, Phys. Rev. B 79, 161407(R) (2009).

[51] S. De Franceschi, L. Kouwenhoven, C. Schönenberger, and W. Wernsdorfer, Hybrid superconductor-quantum dot devices, Nat. Nanotechnol. 5, 703 (2010).

[52] R. Žitko, M. Lee, R. López, R. Aguado, and M.-S. Choi, Josephson current in strongly correlated double quantum dots, Phys. Rev. Lett. 105, 116803 (2010).

[53] A. Martín-Rodero and A. Levy Yeyati, Josephson and Andreev transport through quantum dots, Adv. Phys. 60, 899 (2011).

[54] R. Maurand, T. Meng, E. Bonet, S. Florens, L. Marty, and W. Wernsdorfer, First-Order $0-\pi$ Quantum Phase Transition in the Kondo Regime of a Superconducting Carbon-Nanotube Quantum Dot, Phys. Rev. X 2, 011009 (2012).

[55] B.-K. Kim, Y.-H. Ahn, J.-J. Kim, M.-S. Choi, M.-H. Bae, K. Kang, J. S. Lim, R. López, and N. Kim, Transport Measurement of Andreev Bound States in a Kondo-Correlated Quantum Dot, Phys. Rev. Lett. 110, 076803 (2013).

[56] R. Delagrange, D. J. Luitz, R. Weil, A. Kasumov, V. Meden, H. Bouchiat, and R. Deblock, Manipulating the magnetic state of a carbon nanotube Josephson junction using the superconducting phase, Phys. Rev. B 91, 241401(R) (2015).

[57] R. Delagrange, R. Weil, A. Kasumov, M. Ferrier, H. Bouchiat, and R. Deblock, $0-\pi$ Quantum transition in a carbon nanotube Josephson junction: Universal phase dependence and orbital degeneracy, Physica B 536, 211 (2018).

[58] V. Meden, The Anderson-Josephson quantum dot-a theory perspective, J. Phys.: Condens. Matter 31, 163001 (2019).

[59] J. C. Estrada Saldaña, R. Žitko, J. P. Cleuziou, E. J. H. Lee, V. Zannier, D. Ercolani, L. Sorba, R. Aguado, and S. De Franceschi, Charge localization and reentrant superconductivity in a quasi-ballistic InAs nanowire coupled to superconductors, Sci. Adv. 5, eaav1235 (2019).

[60] S. Baba, J. Sailer, R. S. Deacon, A. Oiwa, K. Shibata, K. Hirakawa, and S. Tarucha, Superconducting transport in single and parallel double InAs quantum dot Josephson junctions with nb-based superconducting electrodes, Appl. Phys. Lett. 107, 222602 (2015).

[61] R. S. Deacon, A. Oiwa, J. Sailer, S. Baba, Y. Kanai, K. Shibata, K. Hirakawa, and S. Tarucha, Cooper pair splitting in parallel quantum dot Josephson junctions, Nat. Commun. 6, 7446 (2015).

[62] B. Probst, F. Domínguez, A. Schroer, A. L. Yeyati, and P. Recher, Signatures of nonlocal cooper-pair transport and of a singlet-triplet transition in the critical current of a doublequantum-dot Josephson junction, Phys. Rev. B 94, 155445 (2016).

[63] L. Hofstetter, S. Csonka, J. Nygård, and C. Schönenberger, Cooper pair splitter realized in a two-quantum-dot Y-junction, Nature (London) 461, 960 (2009). 
[64] A. Das, Y. Ronen, M. Heiblum, D. Mahalu, A. V. Kretinin, and H. Shtrikman, High-efficiency Cooper pair splitting demonstrated by two-particle conductance resonance and positive noise cross-correlation, Nat. Commun. 3, 1165 (2012).

[65] S. Baba, C. Jünger, S. Matsuo, A. Baumgartner, Y. Sato, H. Kamata, K. Li, S. Jeppesen, L. Samuelson, H. Q. Xu, C. Schönenberger, and S. Tarucha, Cooper-pair splitting in two parallel InAs nanowires, New J. Phys. 20, 063021 (2018).

[66] K. Ueda, S. Matsuo, H. Kamata, S. Baba, Y. Sato, Y. Takeshige, K. Li, S. Jeppesen, L. Samuelson, H. Xu, and S. Tarucha, Dominant nonlocal superconducting proximity effect due to electron-electron interaction in a ballistic double nanowire, Sci. Adv. 5, eaaw2194 (2019).

[67] O. Kürtössy, Z. Scherübl, G. Fülöp, I. Endre Lukács, T. Kanne, J. Nygård, P. Makk, and S. Csonka, Andreev molecule in parallel InAs nanowires, arXiv:2103.14083.

[68] T. Kanne, D. Olsteins, M. Marnauza, A. Vekris, J. C. Estrada Saldaña, S. Loric, R. D. Schlosser, D. Ross, S. Csonka, K. Grove-Rasmussen, and J. Nygård, Double nanowires for hybrid quantum devices, arXiv:2103.13938.

[69] See Supplemental Material at http://link.aps.org/supplemental/ 10.1103/PhysRevResearch.3.033240 for methods for analyzing the data and additional measurements.

[70] Additional data as well as raw data used to produce the figures and Supplemental figures from the paper can be found at the repository ERDA of the University of Copenhagen at https://doi.org/10.17894/ucph.9cfe99c5-c548-481cbb19-dd544f53d46a.

[71] S. Guiducci, M. Carrega, F. Taddei, G. Biasiol, H. Courtois, F. Beltram, and S. Heun, Full electrostatic control of quantum interference in an extended trenched Josephson junction, Phys. Rev. B 99, 235419 (2019).

[72] R. Žitko, O. Bodensiek, and T. Pruschke, Effects of magnetic anisotropy on the subgap excitations induced by quantum impurities in a superconducting host, Phys. Rev. B 83, 054512 (2011).

[73] C. W. J. Beenakker and H. van Houten, The superconducting quantum point contact, in Nanostructures and Mesoscopic
Systems, edited by W. P. Kirk and M. A. Reed (Academic Press, Cambridge, MA, USA, 1992), pp. 481-497.

[74] Z. Scherübl, G. Fülöp, C. P. Moca, J. Gramich, A. Baumgartner, P. Makk, T. Elalaily, C. Schönenberger, J. Nygård, G. Zaránd, and S. Csonka, Large spatial extension of the zero-energy $\mathrm{Yu}-$ Shiba-Rusinov state in a magnetic field, Nat. Commun. 11, 1834 (2020).

[75] H. I. Jørgensen, K. Grove-Rasmussen, K.-Y. Wang, A. M. Blackburn, K. Flensberg, P. E. Lindelof, and D. A. Williams, Singlet-triplet physics and shell filling in carbon nanotube double quantum dots, Nat. Phys. 4, 536 (2008).

[76] T. Meng, S. Florens, and P. Simon, Self-consistent description of Andreev bound states in Josephson quantum dot devices, Phys. Rev. B 79, 224521 (2009).

[77] A. Kogan, S. Amasha, D. Goldhaber-Gordon, G. Granger, M. A. Kastner, and H. Shtrikman, Measurements of Kondo and Spin Splitting in Single-Electron Transistors, Phys. Rev. Lett. 93, 166602 (2004).

[78] L. G. Herrmann, F. Portier, P. Roche, A. L. Yeyati, T. Kontos, and C. Strunk, Carbon Nanotubes as Cooper-Pair Beam Splitters, Phys. Rev. Lett. 104, 026801 (2010).

[79] P. Recher, E. V. Sukhorukov, and D. Loss, Andreev tunneling, Coulomb blockade, and resonant transport of nonlocal spinentangled electrons, Phys. Rev. B 63, 165314 (2001).

[80] Z. Scherübl, A. Pályi, and S. Csonka, Transport signatures of an Andreev molecule in a quantum dot-superconductor-quantum dot setup. Beilstein J. Nanotechnol. 10, 363 (2019).

[81] A. Vekris, J. C. Estrada Saldaña, J. de Bruijckere, S. Loric, T. Kanne, M. Marnauza, D. Olsteins, J. Nygård, and K. Grove-Rasmussen, Asymmetric little-parks oscillations in double nanowires, arXiv:2106.01181.

[82] S. Vaitiekėnas, G. W. Winkler, B. van Heck, T. Karzig, M.-T. Deng, K. Flensberg, L. I. Glazman, C. Nayak, P. Krogstrup, R. M. Lutchyn, and C. M. Marcus, Flux-induced topological superconductivity in full-shell nanowires, Science $\mathbf{3 6 7}$, eaav3392 (2020).

[83] F. Peñaranda, R. Aguado, P. San-Jose, and E. Prada, Even-odd effect and Majorana states in full-shell nanowires, Phys. Rev. Research 2, 023171 (2020). 